\title{
Measles in Geneva between 2003 and 2010: persistence of measles outbreaks despite high immunisation
}

\section{coverage}

E Delaporte (Elisabeth.delaporte@etat.ge.ch) ${ }^{1}$, E Jeannot $^{2,3}$, P Sudre ${ }^{1}$, C A Wyler Lazarevic ${ }^{2}$, J L Richard ${ }^{4}$, P Chastonay ${ }^{3}$

1. Epidemiology and Communicable Diseases Section, Cantonal Health Service, General Directorate for Health, Department of Regional Affairs, Economy and Health, Geneva, Switzerland

2. Youth Health Service, Department of Education, Geneva, Switzerland

3. Institute of Social and Preventive Medicine, University of Geneva medical school, Geneva, Switzerland

4. Infectious Diseases Division, Federal Office of Public Health, Berne, Switzerland

Delaporte E, Jeannot E, Sudre P, Wyler Lazarevic CA, Richard JL, Chastonay P. Measles in Geneva between 2003 and 2010 : persistence of measles outbreaks despite high immunisation coverage.

Euro Surveill. 2011;16(39):pii=19980. Available online: http://www.eurosurveillance.org/ViewArticle.aspx?Articleld=19980

Article published on 29 September 2011

Despite high immunisation coverage, several measles outbreaks occurred in the canton of Geneva between 2003 and 2010, with 161 reported cases (52 in 2003, 16 in 2005 and 93 in 2007-2010). It affected mainly 10-14 year-old children in 2003 (31\%), and adults 20 years and older in 2005 (75\%) and 2007-2010 (39\%). Several cases were imported from neighbouring cantons and countries, as confirmed by the diversity of the genotypes identified (D8, D6, D5, D4 and $\mathrm{G}_{3}$ ). Infections were mainly transmitted via family (54\%) and school (22\%) in 2003 , hospital (71\%) and family (29\%) in 2005 , and family (55\%) and school (26\%) in 2007-2010. In 2003, $6 \%$ of infected patients were vaccinated, $27 \%$ in 2005 and $2 \%$ in 2007-2010, none of them with two doses of measles-containing vaccine. Between 2003 and 2008, measles vaccine coverage, particularly for the second dose, increased by 23 percentage points to $91.7 \%$ in the 28 month-olds, by 27 points to $92.3 \%$ in the $5-6$ year-olds, and by 19 points to $86 \%$ in the $13-14$ year-olds. In a cosmopolitan setting where immunisation coverage is high but not sufficient to eradicate measles, outbreaks can be limited by efficient surveillance and early control measures. Catch-up vaccination needs to be reinforced among teenagers and young adults.

\section{Introduction}

In Switzerland, measles immunisation with a single dose of monovalent vaccine has been recommended since from 1976. In 1985, this vaccine was replaced by one dose of the measles-mumps-rubella (MMR) vaccine, and in 1996, a second dose of the MMR vaccine was introduced. Currently, the first dose is recommended at the age of 12 months, and the second at 15-24 months. In the canton of Geneva, measles immunisation coverage in children aged 28 months was $89.7 \%$ for the two vaccine doses in 2007 , after a continuous progression since its introduction [1]. This increase resulted, at least in part, from continuous efforts of physicians to vaccinate infants, health authorities providing regular individual information to parents, and media coverage of local measles-related events. However, several measles outbreaks have been recorded in this canton since 2003 , with a total of 161 cases at the end of 2010. Following two outbreaks in 2003 and 2005, several outbreaks occurred in quick succession between 2007 and 2010 in the wider context of a national epidemic.

Measles eradication is a public health priority at both national [2] and European level. The World Health Organization (WHO) had set the goal of eliminating measles in Europe by the end of 2010 [3], but this has recently been postponed to 2015 [4]. In order to achieve this goal, an immunisation coverage of at least $95 \%$ with two doses is necessary [5]. The immunisation coverage in the Geneva canton approaches this threshold, but it appears still too low and too recent to provide herd immunity so as to stop the transmission of the measles virus [1]. Measles outbreaks regularly occur as a result of the progressive increase, during inter-epidemic periods, of non-immunised people and young adults vaccinated with only one dose, in addition to the regular importation of measles from neighbouring regions.

This article describes the measles outbreaks that occurred in the canton of Geneva between 2003 and 2010 and aims to assess whether an efficient alert system related to early and effective measures is able to control measles outbreaks in a canton where immunisation coverage is suboptimal to eliminate measles.

\section{Methods}

Surveillance of measles cases

In Switzerland, physicians have been required since 1999 to notify within 24 hours to the cantonal health authorities all cases presenting the symptom triad of fever, maculopapular rash, and one or more of the 
following symptoms: cough, rhinitis or conjunctivitis. This initial notification is followed by another, more detailed notification. Similarly, since 1988, laboratories are obliged to notify all confirmed measles cases within 24 hours.

Our analysis was based on measles cases in the canton of Geneva that were notified by physicians and laboratories between 1 January 2003 and 31 December 2010. It also included cases in contacts, defined as cases who did not seek medical consultation and were thus not included in the mandatory notification but instead reported orally by physicians or patients and investigated by the cantonal health authority.

Notification data from suspected cases with a negative laboratory result or post-vaccination measles (one case) were excluded. The analysed cases were classified as clinical or confirmed cases. Clinical measles was defined as the occurrence of a generalised rash associated with fever and one or more of the following symptoms: cough, rhinitis or conjunctivitis. A case was considered confirmed if there was i) a positive laboratory test (measles-specific IgM, RT-PCR, virus isolation or IgG seroconversion/IgG titre increase) and at least one of the aforementioned clinical signs, or ii) clinical measles with an epidemiological link to a laboratoryconfirmed case.

In selected cases, the viral genotype was identified in saliva samples by the Robert Koch Institute, Berlin,
Germany, and, from 2007 onwards, by the Central Laboratory of Virology of the University Hospital of Geneva (HUG).

Imported measles referred to cases where the virological or epidemiological data confirmed that the exposure occurred outside the canton or country during the 7-21 days before onset of the rash [3]. A case linked to imported measles referred to a locally infected patient in the setting of a transmission chain that started with the imported case.

\section{Surveillance of immunisation coverage}

The method used to collect data has been described in detail previously [6,7]. The annual immunisation coverage involved children at the age of 28 months, 5-6 years, and 13-14 years in a given year between 2003 and 2008. After this date, the sampling method changed. The indicator used to analyse the immunisation coverage and its evolution was the proportion of children who received either one or two doses of the measles vaccine. We analysed only data from children whose vaccination records were available and interpretable.

Analysis of measles cases and immunisation coverage of children aged 28 months was conducted using SPSS software for Windows, version 18. For schoolaged children, Stata software, version 10, was used. Comparisons between the groups were performed by means of the chi-squared test; a two-sided $p$ value of

\section{FIGURE 1}

Epidemic curve of measles cases and transmission chains, Geneva canton, 2003-2010 (n=161)
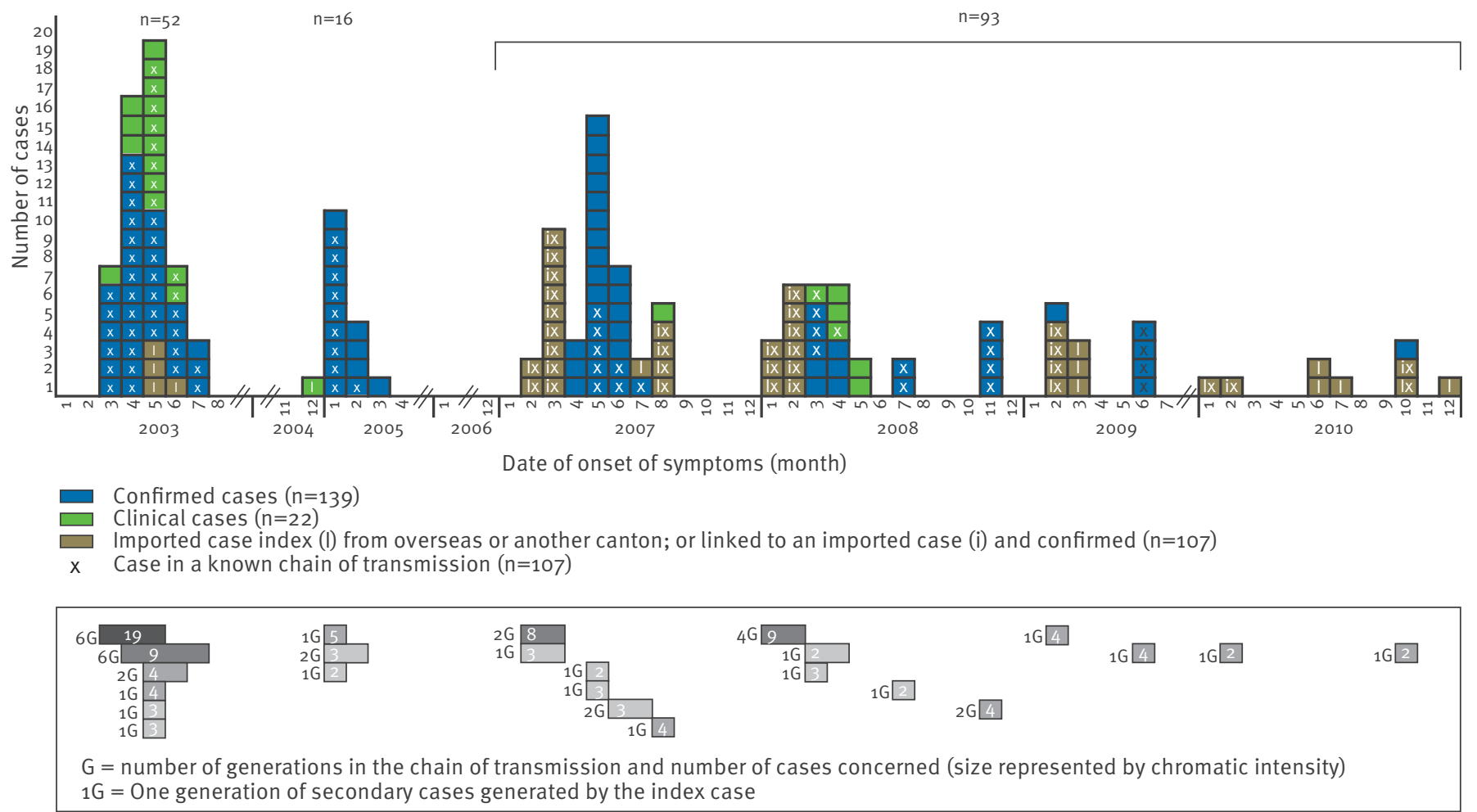
0.05 was considered statistically significant. For the measure of immunisation coverage, $95 \%$ confidence intervals $(\mathrm{Cl})$ were employed.

\section{Results}

\section{Census of the cases}

Between 2003 and 2010, three large measles outbreaks followed one another in the canton of Geneva (453,000 inhabitants in 2009), with a total of 161 cases reported to the General Directorate of Health (Direction générale de la santé, DGS) (Figure 1). The duration and intensity of these outbreaks were variable. In 2003, 52 cases were reported over a period of five months (annual incidence rate of 12.0 per 100,000 inhabitants), while in 2005, 16 cases were reported over four months (annual incidence rate of 3.6 per 100,000 inhabitants). In contrast, the series of outbreaks between 2007 and 2010 was especially long, with 93 cases occurring over 47 months (annual incidence rate of 5.1 per 100,000 inhabitants). After an initial outbreak of 43 cases over five months in 2007 and another one of 23 cases over five months at the beginning of 2008 , several outbreaks with very low case numbers and duration followed, with isolated cases also occurring.

In 2003, 37 confirmed cases were reported (19 laboratory-confirmed and 18 with an epidemiological link to a laboratory confirmation), 16 in 2005 (14 laboratoryconfirmed and two with an epidemiological link), and 86 in 2007-2010 (62 laboratory-confirmed and 24 with an epidemiological link). In 2003, 43 of 52 cases were reported as part of the mandatory notification system, compared with all 16 cases in 2005 and 78 of 93 cases in 2007-2010. The other cases (nine in 2003 and 15 in 2007-2010) were identified by active case finding among patients, their contacts, and physicians' practices.

In 2003 , the median duration between the onset of symptoms (medical consultation date is generally unknown) and receipt of the first written notification by the physician or laboratory was nine days. For the 16 cases occurring in 2005, this duration was 13 days for the first eight cases and 8.5 days for the final eight cases. Comparing the first 10 cases in 2007 with the nine cases in 2010, it decreased from 8 to 6.5 days.

\section{Demographic characteristics}

\section{and laboratory findings}

In 2003, 32 men and boys and 20 women and girls contracted measles whereas 43 men and boys and 50 women and girls were reported in 2007-2010. In 2003, 16 children were aged between 10 and 14 years, whereas 12 adults were aged 20 years or older in 2005 and 36 in 2007-2010. Median age of the infected subjects was 12 years in 2003, 32.5 years in 2005, and 16 years in 2007-2010. In 2005, the majority of cases were adults and there was a slight increasing trend in the subjects' age, which was statistically non-significant (linear regression model).
RNA from 41 saliva samples or throat swabs was analysed and 35 were successfully sequenced (Table). In 2003, the detected viruses belonged to genotypes D8 $(n=3)$ and $D_{5}(n=1)$. In 2005 , the circulating viruses were genotypes D6 $(n=3)$ and D8 $(n=1)$. In 2007, genotype $D_{5}$ predominated $(n=13)$, but genotype $B_{3}$ was also detected $(n=1)$. Genotype $D_{5}$ was identified in $2008(n=3)$ and $2009(n=3)$, and simultaneously with genotype D4 $(n=2)$ in 2009. Finally, in 2010, three different virus genotypes, D4 $(n=1), G_{3}(n=2)$, and D8 $(n=1)$, were found. Moreover, one case of post-vaccination measles (genotype A) occurred 12 days after a post-partum MMR vaccination.

Among the 47 cases for whom the vaccination status was known in 2003 , three had received one dose of the measles vaccine. In 2005, among the 11 cases with known vaccination status, three were vaccinated, whereas in 2007-2010, among the 82 cases with known vaccination status, only two were vaccinated. During these outbreaks, none of the infected subjects

\section{TABLE}

Distribution of measles virus genotypes, canton of Geneva, 2003-2010 ( $\mathrm{n}=35)$

\begin{tabular}{|l|c|c|c|c|c|c|c|c|}
\hline \multirow{2}{*}{ Year } & $\begin{array}{c}\text { Number of samples } \\
\text { tested }\end{array}$ & \multicolumn{6}{|c|}{ Measles virus genotype detected } \\
\cline { 2 - 11 } & B3 & D8 & D6 & D5 & D4 & G3 & A \\
\hline 2003 & 4 & 0 & 3 & 0 & 1 & 0 & 0 & 0 \\
\hline 2004 & 0 & 0 & 0 & 0 & 0 & 0 & 0 & 0 \\
\hline 2005 & 4 & 0 & 1 & 3 & 0 & 0 & 0 & 0 \\
\hline 2006 & 0 & 0 & 0 & 0 & 0 & 0 & 0 & 0 \\
\hline 2007 & 19 & 1 & 0 & 0 & 13 & 0 & 0 & 1 \\
\hline 2008 & 5 & 0 & 0 & 0 & 3 & 0 & 0 & 0 \\
\hline 2009 & 5 & 0 & 0 & 0 & 3 & 2 & 0 & 0 \\
\hline 2010 & 4 & 0 & 1 & 0 & 0 & 1 & 2 & 0 \\
\hline Total & $4^{\text {a }}$ & 1 & 5 & 3 & 20 & 3 & 2 & 1 \\
\hline
\end{tabular}

a Six samples could not be successfully typed.

\section{FIGURE 2}

Cases of measles according to vaccination status, Geneva canton, 2003-2010 ( $\mathrm{n}=161)$

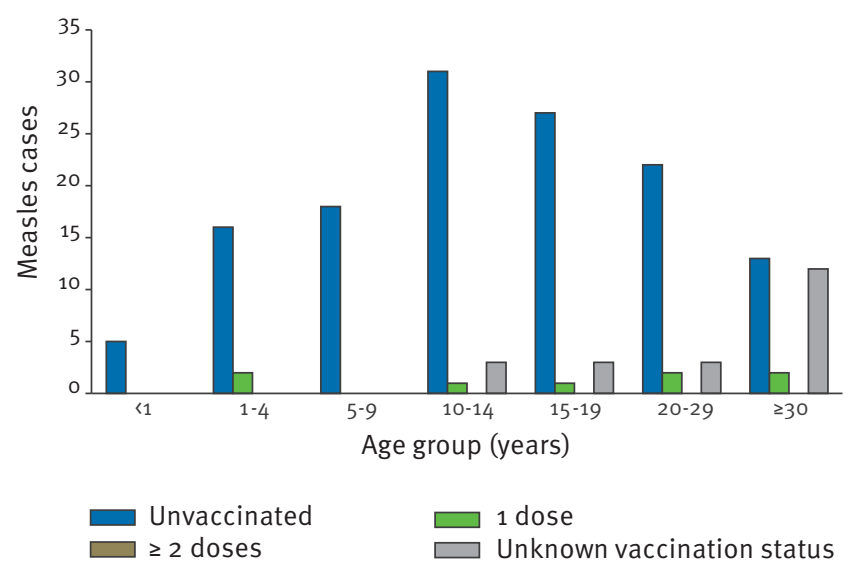


had received a full two-dose vaccination. None of the five under one year-old infants who contracted measles were vaccinated. The proportion of patients whose vaccination status was unknown increased with age (Figure 2).

\section{Chains of transmission}

In 2003, six chains of transmission were identified, comprising 42 of the cases reported in that year. They consisted of two large chains of transmission of six generations following the index case, involving 19 and nine cases, respectively, one chain of two generations (four cases), and three chains of a single generation with four, three, and three cases, respectively (Figure 1). In 2005, three chains of transmission were identified in 10 cases: two chains of a single generation involving five and two cases, respectively, and one chain of two generations with three cases. Finally, 15 chains of transmission ( 55 cases) were observed in 2007-2010: one large chain of four generations involving nine cases, three chains of two generations with eight, four, and three cases, respectively, and 11 chains of a single generation with two to four cases (Figure 1).

Several cases were imported from foreign countries and some of them were related to secondary cases: France in 2003 (one imported case and three secondary cases, unknown genotype), Ethiopia in 2007 (one imported, no secondary, genotype B3) and Germany in 2008 (one imported, eight secondary, genotype D5). Eight of the nine cases occurring in $\mathbf{2 0 1 0}$ were imported or related to imported cases, originating from India (one imported, one secondary, genotype D8), London (one imported, one secondary, genotype G3), Italy (one imported, no secondary, unknown genotype) and France (three imported, no secondary, genotype D4 for one case). Several cases were also imported from other Swiss cantons or related to these importations: Zurich in 2005 (one imported, no secondary, unknown genotype), Berne in 2007 (one imported, seven secondary, genotype $D_{5}$ ), Vaud (one imported, two secondary, unknown genotype) and Ticino (two imported, two secondary, genotype $D_{5}$ ). In 2009, importations from the cantons of Vaud (three imported, four secondary, genotype $D_{5}$ for two cases) and Berne (one imported, no secondary, unknown genotype) were identified. There were no cases imported from other cantons in 2010.

Measles infections were mainly transmitted via family (20 of 37) and school (eight of 37) in 2003, hospital (five of seven) [8] and family (two of seven) in 2005, and family (21 of 38) and school (10 of 38) in 2007-2010. Outbreaks had high transmission rates among unvaccinated siblings and in schools with a philosophy that attracted parents reluctant to vaccinate their children. Several cases of nosocomial transmission were also reported in medical practices attended by parents who refused to vaccinate their children. During the outbreak in 2005 , cases of nosocomial transmission were identified among young non-vaccinated or insufficiently vaccinated healthcare professionals who did not receive catch-up vaccinations after the initiation of the twodose vaccination schedule [8]. There were, however, no cases of transmission by a caregiver.

\section{Complications}

In 2003 , seven of 52 patients developed at least one complication compared with three of 16 in 2005 and 11 of 93 in 2007-2010. Three patients were hospitalised in 2003, four in 2005, and 18 in 2007-2010. The most common complication was pneumonia, which was observed in five of the patients in $\mathbf{2 0 0 3}$, one in $\mathbf{2 0 0 5}$, and seven in 2007-2010. Two patients required admission to the intensive care unit. No encephalitides or deaths were reported.

The median age of patients with complications was 21 years, compared with 14 years for those without complications. The number of patients with complications or requiring hospitalisation increased significantly with age (p<0.05): no infants under one year of age, two children aged 1-4 years, three aged 5-9 years, five aged 10-14 years, six for the 15-17 years and 20 adults aged 20 years or more. The risk of hospitalisation increased even more significantly with age ( $p<0.005)$. No infants or children aged 1-4 years were hospitalised, two children aged 5-9 and two aged 10-14 years, four children aged 15-19 years, and 17 adults aged 20 years or more. The risk of pneumonia was largest for children aged 10-14 years $(n=5)$. The most frequent reason for hospitalisation was poor general health (nine patients, with a median age of 24.5 years), followed by pneumonia (seven patients, median age of 14 years). None of the patients who developed a measles-related complication was vaccinated.

\section{Preventative measures for subjects in} contact with measles-infected patients In 2003, the cantonal health authorities implemented the following measures for people who were in contact with measles-infected patients: i) communication and information of the patient's environment (school, crèche) along with vaccination recommendations, ii) rapid identification of measles-susceptible contacts, considered to be individuals without at least one dose of the measles vaccination, history of measles or serologically-confirmed immunity, and iii) post-exposure vaccination of contacts within 72 hours. Since 2007, an additional measure has been introduced in situations where no post-exposure vaccination was given within a suitable delay. In these situations, the exposed person should avoid contact with susceptible individuals for a period of 18 days following the last exposition to the case.

Since 2003, among the 14 non-immunised contacts who were identified and received post-exposure vaccination (13 siblings of cases and one friend), three did not develop measles. For two of them, the vaccine was administered within 72 hours after exposure, and for the third (who was exposed to an unconfirmed index case), the vaccine was administered on the fifth day. 
Among the 11 contacts who did develop the disease, median duration between the onset of symptoms in the index case and the vaccination was five days (range: 3-6 days).

During the 2007-2010 outbreaks, school exclusion measures were taken for 15 non-immunised contact subjects with clear evidence of exposure, including siblings, a mother (teacher), and a friend. Among them, 13 developed measles during the exclusion period. No secondary cases resulted from 12 of these cases (the situation for the final case is uncertain).

Surveillance of measles immunisation coverage Between 2003 and 2008, a total of 25,390 28-monthold children who were officially registered as residents in the canton of Geneva were eligible for the monitoring of their vaccination records. However, information was unavailable for 2,923 of them. Thus, the analysis was based on 22,467 28-month-olds, which corresponds to an overall response rate of $88.5 \%$. For children aged 5-6 years and 13-14 years, a cross-sectional analysis was repeated in the school years 2003/04 and 2008/09 in these two age groups. All the pupils attending state schools in Geneva were included in this study, but the analysis was only based on those whose vaccination records were completed and interpretable, and had been sent to the Youth Health Department (Service de la santé de la jeunesse - SSJ), which represented 90\% of the total.

Between 2003 and 2008, immunisation coverage of 28-month-olds with one vaccine dose increased from $94.8 \%(95 \% \mathrm{Cl}: 94.1 \%-95.4 \%)$ to $96.1 \%(95 \% \mathrm{Cl}$ : $95.5 \%-96.7 \%)(p=0.002)$ (Figure $3 A)$. For two doses, it strongly increased from $68.9 \%$ (95\% Cl: $67.5 \%-70.3 \%$ ) to $91.7 \%$ (95\% Cl: 90.9\%-92.6\%) (p<0.001) (Figure 3B). For the 5-6-year-olds, immunisation coverage for one vaccine dose increased from $92.7 \%(95 \% \mathrm{Cl}: 91.8 \%-$ 93.5\%) to $96.1 \%$ (95\% Cl: $95.3 \%-96.8 \%$ ) (p<0.001). For two doses, coverage strongly increased from $65.4 \%$ (95\% Cl: $63.8 \%-67.0 \%$ ) to $92.3 \%$ (95\% Cl: $91.3 \%-$ 93.3\%) (p<0.001). Finally, for the children aged 13-14 years, immunisation coverage for one dose increased from $89.7 \%$ (95\% Cl: $88.7 \%-90.6 \%$ ) to $91.1 \%$ (95\% Cl: $90.2 \%-92.2 \%)(p=0.03)$. For two doses, it increased from $67.2 \%(95 \% \mathrm{Cl}: 65.7 \%-68.7 \%)$ to $86 \%(95 \% \mathrm{Cl}$ : $84.8 \%-87.2 \%)$ (p<o.001).

\section{Discussion}

Between 2003 and 2010, 161 measles cases were reported to the General Directorate of Health in Geneva, of whom 25 were detected by active case finding during contact tracing. The actual number of cases in the canton during these eight years is probably higher due to the fact that some infected subjects might not have sought medical attention; furthermore, clinical diagnosis may be difficult in the absence of a history of contagion, and some physicians may omit to notify cases.
The surveillance and alert system was reinforced during the period under analysis, with increasingly earlier notifications made by laboratories and physicians over the years. Moreover, there was a marked reduction in the notification delay between the beginning and end of each outbreak. This improvement of the reaction time, from 9-13 days to 8-6 days or less, is at least partially related to reducing the official notification time frame from one week to 24 hours in 2006 , to the media coverage of successive measles outbreaks, and to increased awareness of healthcare professionals following information from the cantonal health authorities. However, there were some late notifications during every outbreak. The efficiency of the alert system was also illustrated by the early notification of several cases with suggestive symptoms, which turned out not to be measles. Thus, 15 suspect cases were investigated, 13 during the period from 2007 to 2010 , but subsequently excluded due to negative serological results. In a recent publication, the WHO proposed that a the sensitivity of surveillance in countries with a measles elimination target is satisfactory when a minimum of two suspected measles cases per 100,000 inhabitants are identified as non-cases in subsequent (laboratory)

\section{FIGURE 3}

Evolution of measles immunisation coverage in children aged 28 months, 5-6 years, and 13-14 years between 2003 and 2008 in the canton of Geneva $(n=63,189)$

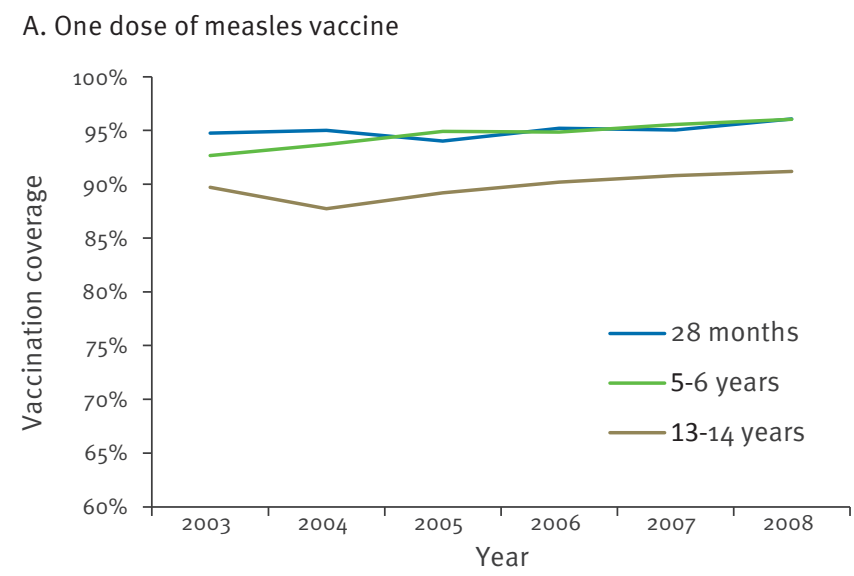

B. Two doses of measles vaccine

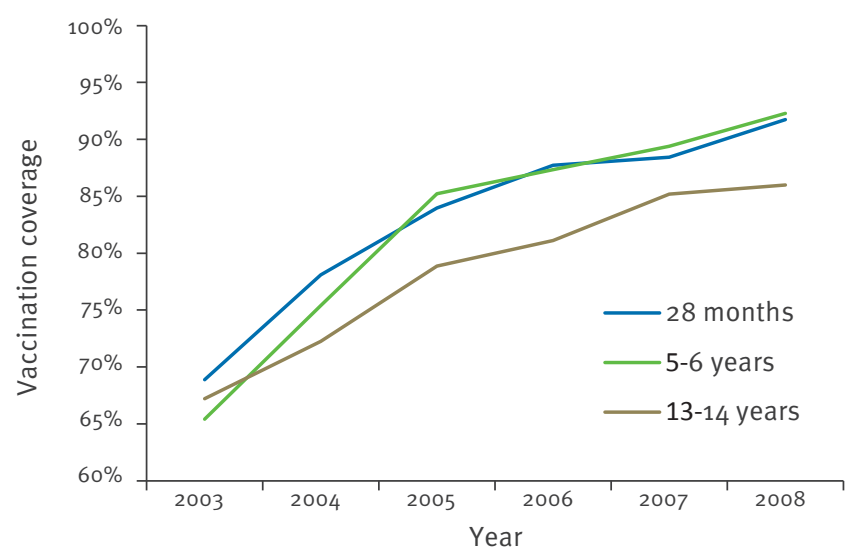


analyses and discarded [9]. In the canton of Geneva, for the period from 2007 to 2010 , the rate was still clearly inferior to the WHO target, with an annual mean of 0.7 discarded cases per 100,000 inhabitants. However, the actual number of discarded cases is certainly higher, considering that the requests to diagnostic laboratory analysis are not currently subject to mandatory notification. Yet, it is not uncommon for physicians to wait for laboratory results and declare measles only if it is confirmed, which is contrary to the legal requirements for mandatory notification.

Control measures have progressively been reinforced since 2003. The identification of non-immunised contacts of measles cases and their vaccination within 72 hours after exposure has become more widespread. School exclusion measures, which did not apply in 2003, became the rule in 2007 for non-immunised siblings of cases. More recently, these measures were extended to all non-immunised contacts in schools and crèches. If rapidly applied, these home quarantine measures are particularly effective given the extreme contagiousness of the virus, and the paramount role played by non-immunised siblings and school transmission in the spread of measles [10]. However, the efficacy of such measures is better still when immunisation coverage is high. In Switzerland, such quarantine is permitted by the federal law on epidemics but not applied equally by the different local health authorities. In Geneva, parents were informed that this was a possibility in case of a school outbreak. Mandatory exclusion from school and nurseries was applied in collaboration with the institutions' directors and, in all instances, well accepted. Most excluded persons developed measles within a few days.

Whereas measles is often regarded as harmless by the general population, $14 \%$ of the patients in Geneva developed at least one complication, and $16 \%$ were hospitalised during the period from 2003 to 2010. During the 2005 outbreak, which affected mainly adults, the proportion of patients with complications or requiring hospitalisation reached $38 \%$. The risk of complications and hospitalisation, or hospitalisation alone, significantly increases with patients' age. Indeed, in adults aged 20 years and older, these risks were $37 \%$ and $30 \%$, respectively. Yet, an increase in the median age of measles cases was observed in the Geneva canton between 2003 and 2010, even though it was small and not statistically significant. At the national level, the proportion of cases aged 20 years and older doubled between the 2003 and 2006-2009 epidemics, increasing from $8 \%$ to $19 \%[11,12]$.

Given this rise in patients' median age, in parallel with the improved immunisation coverage in children and teenagers, the proportion of adult measles cases accompanied by complications and/or hospitalisation is likely to increase. However, regardless of their age, none of the Geneva patients with complicated measles were vaccinated, which indirectly, but reassuringly, confirms that the vaccine effective against measles and its complications. Consequently, catch-up vaccination with up to two doses of the MMR vaccine remains relevant for all measles-naïve subjects, especially adults born after 1963 [13].

Between 2003 and 2008, measles immunisation coverage continuously increased in the three age groups studied. This increase was particularly pronounced for the second dose: an increase by 23 percentage points to $91.7 \%$ for 28 -month-olds, by 27 percentage points to $92.3 \%$ for 5-6-year-olds, and by 19 percentage points to $86 \%$ for $13-14$-year-olds. Thus, in the canton of Geneva, immunisation coverage of young children is currently approaching the $95 \%$ threshold, thus allowing for measles elimination [14]. However, this evolution is rather recent and does not rule out the accumulation of a fairly large number of non-immunised people, particularly among young adults who are no longer exposed to the wild virus (nearly one of five cases was between 30 and 45 years-old in the canton of Geneva).

Based on the immunisation coverage data for dose and age and the notified measles cases, the Swiss Federal Office of Public Health estimated the number of susceptible subjects younger than 20 years to be 8,300 for the canton of Geneva alone, i.e. $8.6 \%$ of this age group [15] (unpublished results), while this proportion is much smaller in adults born after 1963. Subjects born before this date are considered to be immunised due to the widespread circulation of measles during their childhood. Overall, the proportion of immunised subjects in the Geneva population is probably approaching the threshold for herd immunity, at least in part thanks to the clear support and involvement of local physicians. New outbreaks, however, remain highly probable in the case of virus introduction, all the more so because non-immunised subjects largely belong to age groups with strong social interactions: teenagers and young adults.

The number, extent, and duration of measles outbreaks may largely be limited if the surveillance and alert system remains sufficiently effective, early and effective control measures are taken, and immunisation coverage remains at its current level and possibly increases among young adults. Thus, the extent of the transmission chains decreased between 2003 and 2007-2010: in 2003, two chains of transmission of six generations each comprising 19 and nine cases were identified (no exclusion measures were in place for non-immunised subjects), whereas in 2007-2010, chains were mainly limited to two or three cases of intrafamilial transmission owing to post-exposure vaccination and early home quarantine of non-immunised contacts. Moreover, due to one of the best immunisation coverage rates in Switzerland and a routine intervention for each case, the canton of Geneva was one of the least affected districts during the 2006-2009 measles epidemic in Switzerland, with a four-year cumulative incidence rate of 17 per 100,000 inhabitants versus 
57 for the whole of Switzerland, the highest rate of 527 per 100,000 inhabitants being found in the Appenzell Innerrhoden canton [12].

Data relating to the exposure of infected patients together with the genotypic diversity suggest that the introduction of cases from other cantons and from abroad was responsible for some of the outbreaks in Geneva canton. In 2003, the viruses circulating in the Geneva and Schwyz cantons belonged to the genotype D8, but no epidemiological link could be found between these two distant cantons. The main circulating genotype in Switzerland during this period was the $D_{5}$ genotype, which was identified in one Geneva case. Between 2007 and 2009, the D5 genotype (as well as $B_{3}$ and $D_{4}$ in 2009) predominantly circulating in Switzerland was identified in the Geneva canton. In 2010, eight of the nine notified cases were imported from abroad (India, France, Italy, and the United Kingdom) or were related to these importations, with only two very short chains of transmission and three distinct genotypes $D 8, D_{4}$, and $\mathrm{G}_{3}$ ). The $\mathrm{G}_{3}$ genotype [16], which had never previously been identified in Switzerland, was imported from London. Shortly thereafter, another case involving the same genotype with an identical genetic sequence occurred in Geneva, without any known exposure.

Since the summer of 2008 , a change in measles epidemiology has been perceptible in the Geneva canton. Earlier, four outbreaks had occurred that were relatively large (52, 16, 43 and 23 cases) and temporally distant (separated by 16, 22 and four months). Since the summer of 2008 there have been mostly isolated cases and small outbreaks related to imported cases, in relation to a large variety of identified genotypes. The situation observed in 2010 in the canton of Geneva largely corresponds to what is expected after measles elimination, except that with a rate of 6.5 autochthonous cases per million population (i.e. three secondary cases per 463,919 inhabitants), the measles incidence in the canton still exceeds the elimination target of one per million.

These encouraging advances made towards measles elimination on a local level show that determined, politically supported action to promote vaccinations and fight outbreaks do bear fruit. The elimination of measles from Europe by 2015 requires such efforts on a broad scale.

\section{Acknowledgments}

The authors would like to thank Ms. Martine Girard, Brigitte Le Lin, Odile Lacour, Nicole Abbès, and Véra Serena (Epidemiology and communicable diseases section, Cantonal health service, General directorate for health, Department of regional affairs, economy and health, Geneva, Switzerland), Dr Stéphane Hugonnet and Anne Iten (Hospital Infection control Service, Cantonal University Hospital of Geneva, Switzerland) and Pr Claire-Anne Siegrist (Center for Vaccinology and Neonatal Immunology of the Faculty of medicine of the University of Geneva). We also acknowledge the help of the following laboratories and private practitioners: Drs A. Mankertz and S. Santibanez (WHO Regional Reference Laboratory for Measles and Rubella, Robert Koch Institute, Berlin) for genotyping numerous samples, and Drs L. Kaiser, P. Cherpillod, and et S. Cordey from the Central Laboratory of Virology of the University Hospital of Geneva for the RT-PCRs and genotyping of some of the samples. The authors would also like to thank Dr E. Masserey, deputy State Medical Officer of the canton of Vaud and Ms. M. Attinger from State Medical Office of Vaud, as well as the French health authorities from the Departments of Ain and Haute-Savoie for their help at the intercantonal and transborder levels.

\section{References}

1. Federal Office of Public Health. Couverture vaccinale en Suisse 2005-2007. [Vaccination coverage in Switzerland 2005-2007]. Bull OFSP. 2010;11:367-77. French. Available from: http://www. bag.admin.ch/themen/medizin/00682/00685/02133/index.ht ml?lang=fr\&download=NHzLpZeg7t,Inp6loNTU042l2Z6l n1ae2IZn4Z2qZpnO2Yuq2Z6gpJCJdlJ7fWym162epYbg2C _JjKbNoKSn6A

2. Richard JL, Masserey V. Elimination de la rougeole: un objectif national réaliste. [Measles elimination: a realistic national goal]. Bulletin des medecins suisses. 2010;91: 522-4. French.

3. World Health Organization Regional Office for Europe (WHO Euro). Eliminating measles and rubella and preventing congenital rubella infection. WHO European Region strategic plan 2005-2010. Copenhagen: WHO Euro; 2005. Available from: http://www.euro.who.int/_data/assets/pdf_file/0008/79028/ E87772.pdf

4. World Health Organization Regional Office for Europe (WHO Euro). Renewed commitment to elimination of measles and rubella and prevention of congenital rubella syndrome by 2015 and sustained support for polio-free status in the WHO European Region. Resolution EUR/RC60/R12. Copenhagen: WHO Euro; 16 Sept 2010. Available from: http://www.euro.who. int/_data/assets/pdf_file/0016/122236/RC60_eRes12.pdf

5. Anderson RM, May RM. Immunisation and herd immunity. Lancet. 1990;335(8690):641-5.

6. Jeannot E, Wyler Lazarevic CA, Duperrex O, Chastonay P. [Evolution of vaccine coverage from school year 2003-2004 to 2006-2007, for 5 to 6-year-old children in Geneva.] Med Mal Infect. 2010; 40(1): 27-30. French.

7. Jeannot E, Wyler Lazarevic CA, Duperrex O, Chastonay P. [Evolution of the immunization coverage of 13 to 14 year-old adolescents in Geneva between four years]. Santé Publique. 2009;21(6):605-11. French.

8. Hugonnet S, Pittet D, Siegrist C, Vermeulen B, Sudre P, Aramburu C, et al. Rougeole et plan stratégique institutionnel en cas d'épidémie. [Measles and strategic institutional plan in the case of an epidemic]. Swiss-NOSO bull. 2005;12(1):5-8.

9. Suivi des progrès accomplis en vue d'éliminer la rougeole. [Monitoring progress towards measles elimination]. Wkly Epidemiol Rec. 2010;85(49):490-4. French.

10. Delaporte E, Wyler- Lazarevic CA, Richard JL, Sudre P. Contributions des fratries non vaccines à une flambée de rougeole en Suisse. [Contribution of unvaccinated siblings to a measles outbreak in Switzerland]. Rev Epidemiol Sante Publique. 2004;52(6):493-501. French.

11. Richard JL, Zimmermann H. Recent increase in measles in children and teenagers in Switzerland. Euro Surveill. 2003;7(23): pii=2237. Available from: http://www. eurosurveillance.org/ViewArticle.aspx?Articleld $=2237$

12. Richard JL, Masserey Spicher V. Large measles epidemic in Switzerland from 2006 to 2009: consequences for the elimination of measles in Europe. Euro Surveill. 2009:14(50):pii=19443. Available from: http://www. eurosurveillance.org/ViewArticle.aspx?Articleld=19443

13. Office fédéral de la santé publique, Commission fédérale pour les vaccinations. Plan de vaccination suisse 2010. Directives et recommandations. Berne: Office fédéral de la santé publique; 2010. Available from: www.infovac.ch/doc.php? Item $=1 \&$ id $=640$

14. Lang P, Zimmermann H, Piller U, Steffen R, Hatz C. The Swiss national vaccination coverage survey, 2005-2007. Public Health Rep. 2011;126 Suppl 2:97-108.

15. Federal Office of Public Health. Nouvelle vague de l'épidémie de rougeole en début d'année 2009: description et mesures. [New wave of the measles epidemic in early 2009: description and measures.]. Bull OFSP. 2009;27:484-91. Aailable from:html 
?lang=fr\&download=NHzLpZig7t,Inp6loNTU042/2ZZ6In1ae2

IZn4Z2qZpnO2Yuq2Z6gpJCHe31_g2ym162dpYbUzd,

Gpd6emK2Oz9aGodetmqaN19XI2IdvoaCUZ,s-

16. Brown KE, Mulders MN, Freymuth F, Santibanez S, Mosquera

MM, Cordey S, et al. Appearance of a novel measles $\mathrm{G}_{3}$

strain in multiple European countries within a two month

period, 2010. Euro Surveill. 2011;16(17):pii=19852. Available

online: http://www.eurosurveillance.org/ViewArticle.

aspx?Articleld $=19852$ 